\title{
Fundamentos Olvidados de la Política de Defensa: Reflexiones a partir del Caso Argentino
}

\author{
Forgotten Fundamentals of Defense Policy: \\ Reflections as from the Argentinean Case
}

Rev. Bra. Est. Def. v. 2, nº 2, jul./dez. 2015, p. 197-216

ISSN 2358-3932

JORGE BATTAGLINO

Este artículo llama la atención sobre un aspecto de la idea de defensa nacional, entendida tanto como concepto y como política pública que generalmente es dado por sentado en las discusiones sobre el término y que tampoco ha recibido demasiada atención académica (Murray; Viotti, 1994). Nos referimos a sus fundamentos, a aquellos factores que determinan el grado de importancia que un estado le atribuye a la defensa; estos fundamentos, o determinantes, son los que explican el nivel de interés político y social en ella. Este trabajo se ha gestado a partir de la preocupación por lo que podría definirse como la naturalización del interés por la defensa; que implica una lógica de argumentación donde la defensa no necesita ser explicada, que supone que la sola enunciación del término es suficiente para comprender su importancia y lo que se encuentra en juego, que no puede ser otra cosa que la misma supervivencia del Estado (Escudé, 2014). De allí se desprende un postulado normativo, que los políticos deben ocuparse necesariamente de ella, mucho más aún cuando la perspectiva de la guerra, o del conflicto, no ha desaparecido por completo. Sin embargo, la experiencia histórica pone en evidencia que la defensa ha recibido distintos grados de atención y que incluso ha sido desatendida en aquellos casos donde los estados experimentaban la inminencia de la guerra. Algunos trabajos han analizado esta particularidad a partir del estudio de casos en América Latina y en Europa (Pion Berlin; Trinkunas, 2007; Schweller, 2004).

A partir de la indagación de los determinantes de la defensa nacional, el artículo propone una conceptualización de los usos de este concepto. Este trabajo no es un estudio de caso ni tampoco una comparación sino un intento de sistematizar analíticamente cómo debería estudiarse la defensa. Entendemos que las diferentes visiones que existen sobre este tema son un

Jorge Battaglino - Doutor em Política Latinoamericana (Univesity of Essex) e professor de Ciência Política e Estudos Internacionais (Univ. Torcuato di Tella). E-mail: jbattaglino@utdt.edu. 
excelente punto de partida para la delimitación de un campo de estudio. La perspectiva que se propone aquí parte de algunos supuestos conceptuales.

El primero de ellos es que las concepciones esencialistas sobre la defensa, en el sentido de aquellos argumentos que dan por sentada su importancia o que creen que los estados deben preocuparse indefectiblemente por ella, no contribuyen a comprender la riqueza y variaciones históricas que el interés por la defensa tiene en cada unidad estatal. En otras palabras, es una aproximación que dificulta la comprensión de ciertos casos donde ha recibido escasa atención (Schweller, 2004). El interés de las sociedades por la defensa varía considerablemente, aun cuando los estados experimenten amenazas inminentes a su existencia, por ello, su relevancia no debería ser presumida ni naturalizada. La defensa es una dimensión de la política estatal profundamente condicionada por la historia, la cultura, la ideología, la geografía y el contexto regional e internacional; por ello, nunca podría tener el mismo peso para todos los estados.

El segundo supuesto es que a pesar de que la invocación al término defensa nacional pareciera eliminar/suspender cualquier discusión sobre su importancia, la defensa no deja de ser otro concepto de las ciencias sociales y como tal presenta rasgos comunes a algunos de ellos. Se trata de una noción polisémica, aunque generalmente se la asocie con la posesión de mayor o menor cantidad de armamento.

Finalmente, es un concepto disputado en el sentido de que no existe una definición neutral, su orientación tiene efectos sobre la percepción de amenaza de los estados, las misiones de las fuerzas armadas y de seguridad, los recursos que destinan a ellas, como se entrenan o el armamento que adquieren, entre otros aspectos (Frederic, 2011; Pion Berlin; Arceneaux, 2000).

En particular, la experiencia de la Argentina desde 1983 revela con contundencia, y de manera extrema, la importancia de analizar el nivel de los fundamentos. El peso de la visión esencialista en la Argentina se aprecia en el hecho de que la mayoría de los debates sobre el tema gira en torno a cuestiones materiales u organizacionales, como la adquisición de armamento o la cantidad de personal. ${ }^{1}$ Esta aproximación, que reduce la defensa a una discusión sobre los medios (aspectos que obviamente son de suma importancia) no advierte la relevancia conceptual y política de identificar sus determinantes o fundamentos, es decir, aquellos factores que hacen posible que la sociedad y sus políticos se interesen en ella.

Cabe mencionar que la falta de reflexión sobre esta dimensión de la defensa posiblemente se vincule con las características del desarrollo disciplinar de las ciencias sociales. El estudio sobre este concepto no ha ocupado un lugar destacado en las ciencias sociales de América Latina. Los análisis sobre la defensa han sido generalmente marginales si se los compara con 
otras áreas de la ciencia política, las relaciones internacionales o la sociología. Ello se debe a varias razones y entre ellas cabe destacar que el campo de la defensa "es relativamente nuevo" (Murray; Viotti, 1994) y que, además, ha sido incorporado subsidiariamente y opacado por la sofisticación teórica y metodológica de los estudios sobre seguridad (Wilson; Forest, 2008).

Asimismo, la defensa era considerada un asunto reservado a las fuerzas armadas, algo que no alentaba, sobre todo en un periodo de predominio de regímenes militares, el interés académico por estos temas. La llegada de la tercera ola democrática a Sudamérica despertó un fuerte interés por las relaciones civiles militares y, en particular, por los mecanismos e instituciones de control civil de las fuerzas armadas (Diamint, 1999). Esta atracción por el control de los militares abrió paso en la década de 1990, a medida que disminuía la posibilidad de una reversión autoritaria, a la reflexión sobre los temas de defensa. Es allí donde comienza a conformarse una comunidad de académicos en distintos países provenientes de la ciencia política, las relaciones internaciones, la historia, la sociología, entre otras disciplinas, cuyas preocupaciones giraban en torno al campo de los estudios de la defensa tanto en el sentido tradicional del término (amenazas de origen estatal-militar), como de los enfoques que proponían un visión integral subsumiendo la defensa en la seguridad.

Este incipiente campo disciplinar ha alcanzado distintos niveles de institucionalización, siendo Brasil el que más lo ha desarrollado. Sin embargo, se trata de un espacio que presenta una gran diversidad en términos de las concepciones, por ejemplo, respecto a cómo debería estudiarse el concepto o a qué dimensiones debería incluir. El proceso de demarcación del objeto de estudio es inherente a la constitución de cualquier campo disciplinar; y el mismo supone identificar un espacio propio de indagación, una dimensión de la realidad social que tiene especificidad propia. Este ejercicio no se encuentra exento de desafíos sobre todo en vista de la riqueza y diversidad de las lecturas que existen sobre la defensa y, principalmente, por el hecho de que la indagación sobre el objeto de estudio, o sobre el estatus del área como campo de análisis, ha estado ausente.

Este artículo busca contribuir a la demarcación del campo disciplinar proponiendo un enfoque conceptual para estudiar las cuestiones de defensa. Su principal argumento es que esta área puede estudiarse considerando al menos tres niveles de análisis distintos, todos interrelacionados aunque con lógicas diferentes. En primer término, se encuentra el nivel de la definición conceptual de la defensa nacional, generalmente presente en trabajos académicos, pero fundamentalmente en documentos oficiales de los estados. Luego, encontramos el nivel de los medios, es decir, de aquellos aspectos materiales y no materiales que la posibilitan, por ejemplo, la can- 
tidad de armamento que posee un estado, su doctrina militar o si posee industria militar. Por último, existe la indagación sobre aquellos factores que determinan un mayor o menor interés en ella. Los estados destinan más o menos presupuesto, compran más o menos equipamiento, en suma, su interés difiere considerablemente. Esta dimensión, que definimos aquí como los fundamentos de la defensa es, a nuestro juicio, la más decisiva, pues define los recursos materiales y simbólicos que un estado le destinará. En otras palabras, se trata del nivel que permite anticipar cuán viable o sustentable es la defensa de una nación. Estos tres niveles responden a los siguientes interrogantes: ¿Cómo define un estado a la defensa nacional? ¿Cuáles son los medios que despliega para garantizarla? y ¿Cuáles son los factores que determinan el grado de atención política que recibe?

Cabe destacar que el artículo no pretende efectuar una explicación exhaustiva de las definiciones existentes sobre la defensa, ni de los medios asociados y ni de aquellos fundamentos que incentivan o alejan el interés político por ella, por el contrario, la principal motivación es evidenciar la complejidad del concepto y proponer una forma de sistematizar el estudio del mismo. El trabajo asume también una perspectiva normativa al plantear que el nivel de los fundamentos es el que define el grado de atención que la defensa recibirá de un estado. En este sentido, las demandas sociales, políticas o de los mismos militares para incrementar el presupuesto militar o para la adquisición de nuevo equipamiento sólo serán viables si existe interés político para satisfacerlas. Por ello, el análisis de los determinantes es uno de los aspectos que explica la fortaleza o debilidad de la defensa de un estado. Sostener que el interés de los políticos es el que finalmente define los recursos simbólicos y materiales que recibirá la defensa puede parecer evidente, sin embargo, la literatura sobre este tema es escasa. Este artículo se divide en tres secciones principales: en la primera se explica la importancia heurística del caso argentino en términos de la clasificación propuesta; luego se analizan consecutivamente el nivel de las definiciones, el de los medios y finalmente el de los fundamentos.

\section{EL POTENCIAL HEURÍSTICO DEL CASO ARGENTINO}

La defensa en Argentina ha recibido escasa atención de los políticos desde el regreso de la democracia en 1983. Ello se evidencia en la constate disminución del gasto militar, las escasas compras de armamento y en el desmantelamiento de la industria militar durante la década de 1990. A pesar de que esta situación comenzó a revertirse en los últimos años del kirchnerismo, los avances logrados no han sido suficientes para modificar la caracterización de la Argentina post 1983 como un caso de "déficit de 
atención” de las cuestiones de defensa (Pion Berlin; Trinkunas, 2007). En este sentido, se trata de un ejemplo ideal para aislar aquellos factores que favorecen o afectan el interés de una nación en esta área, es decir, para identificar sus fundamentos. El caso es aún más relevante no sólo porque la Argentina mantiene un conflicto territorial con una potencia como Gran Bretaña, con la que mantuvo una guerra en 1982, sino también porque sus vecinos han adquirido importantes cantidades de armamento.

En este sentido, la experiencia de la Argentina pone en evidencia que la defensa no es un concepto que pueda definirse en términos esencialistas; por ello es que las declamaciones sobre su relevancia, sobre el estado de indefensión del país o sobre la obsolescencia del armamento disponible, no logran explicar porqué el interés político continua siendo bajo pese a un panorama que para muchos pone en juego la defensa del país (Calle, 2007).

El desinterés por la defensa en la Argentina comenzó luego de la caída de la última dictadura militar (1976-1983). Cabe destacar que no se trató de un gobierno militar más, de los numerosos que tuvo Argentina durante el siglo XX, sino de uno que provocó la mayor tragedia humana de toda la historia del país y que fracasó en los planos político, económico y militar. El panorama desolador que heredó el presidente Raúl Alfonsín en 1983 condujo previsiblemente a un profundo y persistente divorcio entre la sociedad y todo aquello relacionado con el mundo militar, que ha perdurado hasta la actualidad. Si la sociedad civil había sido definida como "militarista" hasta los años setenta, la experiencia con la última dictadura la volcó al polo contrario, al del antimilitarismo más acérrimo. La defensa no permaneció indemne a esta transformación cultural, identificada de inmediato con lo militar, fue una víctima menor y predecible de la debacle generalizada del régimen militar (Battaglino, 2013).

Cabe mencionar que las cuestiones de defensa habían experimentado una etapa de auge que se extendió durante gran parte del siglo XX y que entró en decadencia luego de la derrota en la guerra de las Malvinas por las razones mencionadas (Battaglino, 2013). Hasta entonces, Argentina destinaba un porcentaje considerable de su presupuesto nacional al gasto militar, que era legitimado por la presencia de tres hipótesis de conflicto simultáneas: dos con países sudamericanos (Brasil y Chile) y otra con una potencia extra-regional (el Reino Unido). Además, el país poseía una importante industria bélica. Esta etapa de apogeo no puede escindirse del papel político que asumieron las fuerzas armadas desde el golpe de estado de 1930. Décadas de tutelaje y de gobiernos militares habían deformado su papel al favorecer una desmedida concentración de poder económico, territorial y organizacional, que luego colapsaría por su propio peso e ineficiencia. 
La derrota en las Malvinas, y la transición por derrumbe que provocó de inmediato, favoreció la retirada desordenada de las fuerzas armadas del gobierno dejándolos en una situación de debilidad política que propiciaría el juzgamiento de militares acusados por violaciones a los derechos humanos y el establecimiento de políticas de control civil inéditas en la historia argentina (Fontana, 1990). La institución militar, mientras tanto, experimentaba una profunda crisis de identidad y fuertes divisiones inter e intrafuerzas que provocaron tres levantamientos durante la presidencia de Raúl Alfonsín (1983-1989). Al mismo tiempo, la sociedad descubría con espanto y asombro la magnitud y lo sistemático de las violaciones a los derechos humanos cometidas desde el aparato del Estado. Éste es el contexto que llevaría a la ruptura civil-militar más importante de la historia argentina; la relación de la sociedad con la defensa nunca volvería a ser la misma.

No es casual, entonces, la pérdida de interés de los políticos argentinos por los temas de defensa: se trata de una conducta racional, cualquier mención a ella era identificada de inmediato con lo militar y provocaba un enérgico rechazo del electorado. La oposición generalizada de la sociedad hacia las fuerzas armadas se convirtió en un fuerte incentivo político para la desactivación de una de las principales fuentes de poder interno que aún mantenían; nos referimos a los conflictos limítrofes pendientes en los años ochenta (López, 1994). Los políticos entendieron que la defensa definida en términos clásicos (la protección contra amenazas externas estatales) era un área funcional para el mantenimiento del poder militar. La presencia de hipótesis de conflicto constituía una fuente de poder organizacional, porque las amenazas percibidas permitían justificar fuerzas armadas de mayor tamaño y presupuesto (Buzan; Weaver, 2003). Así, el mantenimiento de varios escenarios de conflicto bélico distorsionaba la asignación de recursos en un entorno en el que era necesaria su redistribución hacia otras áreas del Estado.

En este marco, el gobierno de Raúl Alfonsín avanzó en la resolución de los problemas limítrofes pendientes con Chile, e inició una etapa de profundización de la cooperación e integración con Brasil. La exitosa distensión que resultó de tal estrategia permitió disminuir considerablemente el presupuesto militar del 3.5\% del PBI en 1983 al 1.8\% en 1989 (Fontana, 1990). Asimismo, la nueva democracia argentina dio un importante paso al sancionar, en 1988, la Ley de Defensa que distingue taxativamente la defensa nacional de la seguridad interior y que limita el empleo de las fuerzas armadas a las agresiones de origen militar, estatal y externo. La ley prohíbe, por lo tanto, el empleo de los militares en misiones de seguridad interna. Sin embargo, el temor a fortalecer nuevamente a la institución militar condujo a que la separación entre defensa externa y seguridad interior, fundamental para un país que había experimentado 50 años de 
golpes militares, no estuviera acompañada por una planeación estratégica que identificara los posibles escenarios de empleo del poder militar en el sentido clásico del término. Esta etapa de declinación de las cuestiones de defensa no se alteró sustancialmente durante los años noventa. La instauración de un programa de ajuste neoliberal reforzó la desatención al reducir aún más el presupuesto de la defensa al 1.1\% del PBI. Asimismo, la industria militar experimentó un brutal ajuste: el 90\% de las empresas de este sector fueron privatizadas o directamente cerradas, y las pocas que sobrevivieron en manos del Estado vieron sus actividades paralizadas o con niveles de producción muy disminuidos (Huser, 2002). Por otra parte, la participación en operaciones de paz se transformó en la principal misión de las fuerzas armadas, a pesar de la importancia de estas misiones, el empleo de los militares en papeles tradicionales continuaba sin ser definido por las autoridades políticas (Anzelini; Poczynok, 2014).

La mayor parte de los especialistas comparten el diagnóstico de que el principal problema de la defensa en la Argentina radicaba en el bajo presupuesto militar, la falta de renovación del equipamiento militar o el problema de gran cantidad de oficiales en relación a los soldados (Acuña; Runza, 2006; Calle 2007; Laferriere; Soprano 2015; Scheetz, 2015). Sin embargo, ninguno de ellos ha hecho referencia a la dimensión de los fundamentos y al hecho de que los mismos han desalentado el interés político y social en ella.

En otras palabras, el cambio drástico en la ideología predominante de la sociedad y de los políticos, que se plasmó en un generalizado rechazo al papel político que los militares cumplieron entre 1930 y 1983, redujo sustancialmente el interés por la defensa y, más importante aún, llevó a una exclusión de la defensa de la estrategia de desarrollo del país.

Por ello, era previsible que las discusiones y los reclamos permanentes sobre la necesidad de adquirir armamento o de otras reformas necesarias luego de 1983 no condujeran a ningún resultado, porque tales demandas no estaban relacionadas con el nivel de los fundamentos, algo que no era contemplado ni por el discurso político como tampoco por el de los especialistas.

\section{DEFENSA NACIONAL: DEFINICIONES OFICIALES Y CONCEPTUALES}

Un primer nivel de análisis de la defensa nacional abarca el conjunto de definiciones que pueden encontrarse en documentos oficiales y en investigaciones académicas. En ambos casos se trata de enunciados abstractos que suelen compartir, sin embargo, dos puntos en común. El primero relacionado con la misma idea de "defensa" que siempre alude al resguardo o protección de ciertos objetos referentes como la soberanía, el territorio, los intereses nacionales, la independencia, la autodeterminación, la integridad 
territorial, los derechos y libertades de los ciudadanos, entre otros aspectos (RESDAL, 2015). El segundo componente se vincula con la protección de tales objetos referentes frente a amenazas que puede ser de origen externo o interno o ambas (Buzan; Waever; Jaap de Wilde, 1998).

En el caso de las definiciones oficiales, la defensa es concebida en términos integrales, como una dimensión de la acción del estado que demanda del esfuerzo coordinado de toda la sociedad. Desde el punto de vista académico se trata de un área de indagación sobre aquellos conflictos, amenazas o riesgos que involucren actual o potencialmente el empleo de las fuerzas armadas en el marco del esfuerzo coordinado de una nación; por ello, como objeto de estudio tiene una lógica integral. Aunque el componente militar tiene un rol central en enfrentar o disuadir riesgos o amenazas, la defensa también se construye y fortalece a partir de la acción de componentes no militares del estado, por ejemplo, de una política exterior que contribuya a fortalecer la resolución pacífica de los conflictos. De allí se desprenden numerosos temas de investigación, desde los más directamente vinculados con el conflicto interestatal como la guerra, la estrategia militar o las alianzas militares, hasta los relacionados con la dimensión no militar de la defensa, como el grado de cohesión social, el nivel de legitimidad social de las fuerzas armadas, la importancia de la industria militar, el papel jugado por los científicos, el rol social de los militares, entre muchos otros.

De esta definición se deduce que el estudio de la defensa es eminentemente interdisciplinar y multidisciplinar al incluir entre otras a la ciencia política, la sociología, las relaciones internacionales, la historia, el derecho, la geografía, los derechos humanos, la geopolítica, los estudios estratégicos, los estudios sobre seguridad internacional. Al mismo tiempo se trata de un área de estudio que no es autónoma, es decir, no posee leyes propias; tampoco es autosuficiente, en el sentido que se baste para explicarse a sí misma, su causa primera, por el contrario, es la política.

Sin embargo, las definiciones oficiales y conceptuales suelen excluir referencias a los medios o instrumentos para alcanzarla protección de los objetos referentes, más allá de las alusiones obvias a las fuerzas armadas como instrumento principal de la defensa de un país. Todos aquellos medios que coadyuvan a que la defensa sea viable o efectiva generalmente no son examinados en este nivel.

\section{DEFENSA NACIONAL: LOS MEDIOS}

No hay defensa nacional viable sin medios adecuados; sin lugar a dudas este nivel es el más mencionado cuando se piensa en la protección de una nación. Si los objetos referentes pueden ser muy variados, lo mismo sucede 
con los medios que suelen ser son los siguientes: las armas, la estrategia/ doctrina militar y las alianzas. Es evidente que la defensa más sólida es aquella que descansa en la presencia simultánea de estos factores, es decir, en el despliegue de fuerzas armadas con capacidad disuasiva, de una estrategia/doctrina militar que se derive lógicamente de objetivos políticos factibles y de un sistema de alianzas que compense las debilidades de un estado.

Es muy común que la defensa nacional se asocie de manera automática con la posesión de armamento en calidad y cantidad (Butfoy, 1997) y, desde esta perspectiva, los instrumentos materiales que la posibilitan asume un rol central: no existe defensa viable sin armas que la hagan posible. Esta perspectiva es muy común en distintas vertientes del realismo político y ha sido difundida en las relaciones internacionales por el enfoque realista en sus diversas variantes. Los supuestos teóricos de la anarquía, la supervivencia estatal y el principio de autoayuda conducen de manera natural al dilema de seguridad y las carreras armamentistas. La imposibilidad de conocer las intenciones últimas de los estados no hace más que perpetuar la dinámica de desconfianza y competencia militar que por momentos puede estar atenuada pero que no puede ser eliminada. Esta lógica lleva a valorar la posesión de capacidades materiales, en particular, las armamentistas que son las que garantizan la supervivencia del estado. La continuidad estatal sólo puede ser asegurada alcanzando el equilibrio de fuerzas militares; de lo contrario, la ventaja militar puede ser explotada de forma oportunista en favor del actor estatal que la posea. De allí se desprende el principio de emulación, que no es más que la constatación de que las naciones suelen incorporar el armamento y la doctrina de los países más exitosos desde el punto de vista militar (Waltz, 1979).

Es indudable que la posesión de armamento es un aspecto de suma importancia para la defensa nacional; sin embargo, la idea de que las armas son las que finalmente deciden el resultado de un conflicto ha sido puesta en duda. En los últimos años autores como Biddle (2006) o Lieber (2005) han sostenido que los estados que poseen las fuerzas armadas más grandes y mejor equipadas no siempre ganan las guerras en las que se embarcan. Más aún, Biddle sostiene que la superioridad material o tecnológica no es lo que garantiza la victoria sino, sobre todo, la doctrina y la táctica empleadas y lo más relevante es la capacidad para eludir o neutralizar las fortalezas del adversario y maximizar las propias. En otras palabras, los resultados de los combates dependen más de la estrategia y táctica militares que del número de tropas o de la superioridad tecnológica. De hecho el actor más débil desde el punto de vista militar fue el que triunfó en el $55 \%$ de las guerras en el periodo 1950-1998 (Arreguin-Toft, 2005). En este sentido, se afirma que la revolución de los asuntos militares, que está 
detrás de las transformaciones de las fuerzas militares de los EEUU, ha sido sobrevalorada (Biddle, 2006).

El debate sobre la importancia de las armas versus la capacidad para concebir la estrategia y la táctica adecuadas, revela la importancia de la dimensión no armamentista de la defensa. Las maquinarias militares más perfectas fueron derrotadas primero en los escritorios de los estrategas. De allí la importancia de este nivel de análisis. Colín Gray (1999) sostiene que la estrategia es el uso que se hace de la fuerza y de la amenaza de ella para los fines de la política, es lo que une política y fuerza; es decir, los objetivos de la política y la estrategia deben estar alineados. La concepción estratégica de un estado parte de analizar su posición en el sistema mundial, de identificar los principales problemas internacionales, cómo afectan a sus intereses, cuáles son sus prioridades, sus aliados y sus valores en política exterior. El nivel estratégico establece el papel que se le asigna a la defensa en el marco de la política exterior, en particular, qué nivel de fuerza militar se está dispuesto a utilizar, en qué tipos de conflictos se debería intervenir y en cuáles abstenerse, los escenarios donde podría actuar y el tipo de relación con las instituciones multilaterales de seguridad.

Las armas y las ideas son medios que permiten alcanzar la defensa de una nación; sin embargo, las alianzas entre unidades del sistema internacional también han sido una herramienta en la búsqueda de maximizar la defensa. El principal objetivo de las alianzas es la protección del interés nacional cuando se percibe que el mismo no puede garantizarse unilateralmente (Walt, 1985). En las relaciones internacionales realistas y liberales han desarrollado distintos argumentos sobre este tema.

Para los realistas la defensa nacional puede garantizarse mediante dos tipos de política de alianzas: la del balance o la del plegamiento. Las políticas de balance tienden a buscar el equilibrio de fuerzas militares a través de un reforzamiento defensivo. Los Estados forman alianzas para enfrentar desequilibrios reales o eventuales, provocados por unidades estatales superiores en capacidades. En el balance puede anticiparse políticas de fortalecimiento de la dimensión militar, la magnitud del mismo será distinta dependiendo de la versión del realismo de la que hablemos. El plegamiento, en cambio, supone una aproximación distinta a la defensa. Ocurre cuando un estado se alinea con otro que es más poderoso, es una estrategia empleada generalmente por los estados débiles. Estas unidades deciden que el costo de oponerse al más fuerte, generalmente próximo geográficamente, excede los beneficios que se pueden alcanzar si no se lo confronta. En los casos de plegamiento la defensa se ve severamente afectada ya que el estado que se pliega acepta su debilidad militar. La supervivencia estatal queda garantizada en este tipo de estrategia porque el más poderoso se 
compromete a respetar la soberanía de aquellos que aceptan su posición en el sistema (Walt, 1985).

Finalmente, los enfoques liberales, como la teoría de la paz democrática, el pacifismo comercial o el institucionalismo, contemplan un sistema internacional donde la difusión de la democracia, el comercio y las instituciones alejen cada vez más la posibilidad de la guerra. Los mecanismos que se postulan son muy variados, desde las restricciones institucionales de la democracia, pasando por las coaliciones económico-sociales transnacionales interesadas en mantener y profundizar el comercio, hasta el potencial de las instituciones internacionales para generar compromisos y reciprocidad. De este modo, algunos países entienden que la mejor estrategia para garantizar la defensa nacional es aquella que descansa en la profundización de estos procesos, en otras palabras, en la difusión de la democracia, en la expansión del comercio mundial y en el fortalecimiento de las instituciones y regímenes globales (Oneal; Russett, 1997).

\section{DEFENSA NACIONAL: SUS FUNDAMENTOS}

La dimensión de los fundamentos de la defensa nacional ha sido virtualmente olvidada tanto en el debate público como en el análisis académico sobre el tema. La discusión e indagación sobre ella suele concentrarse casi exclusivamente en el nivel de los medios y, en particular, en torno a la cantidad y calidad del armamento que un estado posee o a cuestiones de estrategia y táctica militar. Sin embargo, este tipo de aproximación no permite avanzar sobre la comprensión de ciertos fenómenos claves, como por ejemplo, los motivos por los que los países destinan más o menos recursos a sus fuerzas armadas defensa. En este sentido, el nivel de los fundamentos gira en torno a la identificación y explicación de las condiciones políticas que incentivan, o no, el interés de los políticos y la sociedad en la defensa nacional. Desde el punto de vista metodológico los fundamentos permiten dar cuenta de las variaciones en los medios, es decir, son su variable independiente.

Cabe aclarar que la literatura sobre el tema es escasa y para el caso de América Latina se reduce al trabajo de Trinkunas y Pion Berlin (2007) quienes proponen el concepto de "déficit de atención" como una situación en la que la sociedad civil y política tienen un bajo interés por la defensa nacional en el sentido de que recibe escasos recursos materiales y simbólicos. Estos autores sostienen que existen factores estructurales y sociales que determinan el grado de interés; de este modo, este trabajo señala la importancia de identificar y analizar aquellos aspectos que explican la fortaleza o debilidad de la defensa de una nación. 
La importancia de este nivel fue señalada con anterioridad en El Soldado y el Estado, el clásico trabajo de Huntington (1995) sobre relaciones civiles militares. Aunque allí no se plantea abiertamente la cuestión de los fundamentos, el análisis de Huntington sobre el patrón histórico de las relaciones civiles militares en los Estados Unidos reconoce implícitamente la relevancia explicativa del nivel de los determinantes para explicar la viabilidad de la defensa nacional. Este aspecto se desprende del análisis de los imperativos funcionales y sociales.

Según Huntington, el equilibrio de las relaciones civiles militares, que son esencialmente conflictivas desde su perspectiva, depende de la coexistencia armónica de dos variables: los imperativos sociales y los imperativos funcionales. Los imperativos sociales se derivan de la ideología predominante de la sociedad. Huntington identifica cuatro grandes ideologías: liberal anti-militar, conservadora pro-militar, fascista pro-militar y marxista anti-militar. Por otra parte, los imperativos funcionales se derivan de las amenazas externas percibidas; en este sentido, el grado de compatibilidad entre la ideología predominante y el nivel de amenaza externa es la clave explicativa de la fortaleza o debilidad de la defensa nacional. Huntington analiza el caso de los EEUU hasta la década de 1950 para sustentar su argumento. Sostiene que hasta la Segunda Guerra Mundial la ideología predominante fue profundamente antimilitarista y que este imperativo social permitió, en un contexto de baja amenaza externa, un bajo nivel de gasto militar y de despliegue permanente de militares. En efecto, hasta el comienzo de la Segunda Guerra la sociedad estadounidense había tenido una visión negativa de sus militares; los mismos eran vistos con sospecha y hostilidad. Se consideraba que las fuerzas armadas eran solamente necesarias en tiempos de crisis, cuando la sociedad reconocía la necesidad de movilizar su poder militar pero, cuando el peligro se conjuraba, se aislaba y debilitaba a los militares hasta que surgiera un nuevo conflicto. La Guerra Civil, el conflicto Hispano-Americano y la Primera Guerra Mundial fueron claros ejemplos de este patrón que revelaba el rechazo de la sociedad estadounidense a la presencia de aparatos militares importantes.

El enfoque de los imperativos permite que Huntington pueda anticipar cuándo es más probable que se produzca una crisis en la defensa nacional. Desde esta perspectiva, la defensa de los Estados Unidos enfrentará una etapa de riesgo si se modifica el imperativo funcional (incremento del nivel de amenaza externa) en el contexto de un imperativo social que permanece inalterado. Este es el escenario que Huntington describe al cabo de la Segunda Guerra Mundial cuando la amenaza soviética se vuelve permanente. Existen dos posibles resultados en un contexto de asincronía de los imperativos: o la ideología permanece inmune, lo cual pondría en riesgo a 
la nación, o la sociedad se vuelve más conservadora de manera de enfrentar la amenaza externa. Huntington se inclina por esta segunda opción, pero más allá de su posición normativa, lo relevante de su análisis es que identifica el que quizás sea el principal dilema en el nivel de los fundamentos: cómo compatibilizar las necesidades de la defensa con los valores e ideología dominantes de una sociedad. La respuesta a este dilema es de vital importancia para la protección de un estado y no puede avanzarse en ella sin reconocer la relevancia de este nivel de análisis cuando se diseña e implementa la política para el área.

En suma, la teoría de Huntington contribuye a señalar la importancia de dos de los principales fundamentos: el nivel de amenazas y los efectos que ejerce sobre la defensa/fuerzas armadas la ideología predominante de una sociedad.

La percepción de amenaza que experimenta un país es un incentivo fenomenal para que los políticos y la sociedad se interesen por la defensa. Los estados que experimentan altos niveles de riesgo externo son los que suelen destinar mayor atención material y simbólica a su dispositivo militar (Desch, 1996). Por el contrario, los países que no enfrentan tales amenazas y que perciben que su supervivencia se encuentra garantizada suelen experimentar "déficit de amenazas".

Asimismo, existen otras lecturas sobre el impacto de la ideología de las elites políticas y de la sociedad en el grado de interés por la defensa. Aunque se suele partir del supuesto de que la ideas conservadoras o de derecha son más favorables a la defensa nacional, como Huntington sostenía, mientras que las de izquierda tienden a rechazar altos presupuestos militares o políticas exteriores agresivas, en los hechos tal diferencia no se verifica con tanta precisión. El caso de la Argentina es un claro ejemplo de este punto ya que durante la década de los 1990 la implementación del programa neoliberal fue funcional a una continua reducción del presupuesto militar y al desmantelamiento de la industria militar (Eissa, 2010). De hecho, la agenda de la defensa ocupó un lugar marginal durante ese periodo. Ello se debió a que el modelo de desarrollo que proponía el neoliberalismo se asentaba en una lectura del mundo fundada en los principios de la globalización liberal en la que la soberanía y la autonomía estatal parecían principios que se derrumbaban. Organizar un sistema de protección efectivo con el fin de enfrentar potenciales conflictos con fuerzas armadas de otros estados, carecía de sentido cuando se confiaba en el poder pacificador del comercio y las alianzas internacionales para garantizar la soberanía. Este discurso de relativización de la soberanía, sumado a la implementación del Consenso de Washington, condujo al desmantelamiento de la industria militar y a la cancelación de desarrollos tecnológicos de avanzada que eran 
banalizados y acusados de ineficientes desde el punto de vista económico y comercial. No es de extrañar, entonces, que las misiones de paz se transformaran, virtualmente, en la misión principal para los militares y que la doctrina de las nuevas amenazas tuviera tanta difusión por aquellos años; algo coherente para un modelo de desarrollo que concebía a la defensa como un gasto innecesario y que tendía a definir al rol militar en términos crecientemente policiales (Canelo 2011).

Por otra parte, aunque la izquierda ha sido definida en muchas ocasiones como antimilitarista, sobre todo como resultado de sus históricos conflictos con las fuerzas armadas, la experiencia reciente de los gobiernos de la nueva izquierda sudamericana muestra el patrón contrario: de un acercamiento a la defensa y a las fuerzas armadas que ha repercutido favorablemente en el aumento del gasto militar y en la adquisición de armamento (Battaglino, 2015). De hecho, estos gobiernos han implementado proyectos políticos que atribuyen a la defensa y/o a las fuerzas armadas el rol de actores claves para alcanzar objetivos definidos como estratégicos. Las administraciones de la nueva izquierda han implementado modelos de desarrollo que han incluido a la defensa/fuerzas armadas; en particular, en el marco de la implementación de distintas variantes de programas neodesarrollistas (D’Araujo, 2010). Los militares también han sido incorporados cuando las capacidades estatales civiles no son suficientes para implementar políticas de ampliación de derechos en vastos sectores de la población. Tal es el caso de Bolivia y en menor medida de Brasil (Dube; Pinzon, 2012).

En este sentido, quizás sea necesario considerar no sólo la orientación ideológica de un gobierno sino también su modelo de desarrollo y, sobre todo, en qué medida incorpora a la defensa/fuerzas armadas para el cumplimiento de sus objetivos. La relevancia de éstos pueden aumentar cuando el modelo de desarrollo incluye objetivos tales como incrementar los márgenes de autonomía nacional para el manejo de los recursos definidos como estratégicos o para mejorar el control de su territorio, en estos casos se suele valorar el despliegue de un instrumento militar debidamente equipado. La importancia atribuida a la autonomía también queda en evidencia en los programas neodesarrollistas que perciben a la defensa como un motor del desarrollo económico y también de su garantía y protección, este aspecto estuvo presente en el diseño de la estrategia de defensa de Brasil del 2008 (D’Araujo, 2010). Desde esta perspectiva, una protección adecuada permite garantizar la soberanía y la integridad territorial, que son los pilares esenciales para el desarrollo y la protección de todos los recursos materiales y humanos que posee la nación. En otros casos, el motor de la defensa lo constituye la implementación de estrategias de política exterior 
que incluyen la posibilidad de desplegar globalmente su poder militar, lo cual demanda de una organización equipada para poder interoperar en misiones multilaterales con las fuerzas armadas más avanzadas del planeta.

Asimismo, el antimilitarismo de los políticos y de la sociedad no se encuentra solamente determinado por su ideología sino también por experiencias traumáticas o conflictivas con las fuerzas armadas; tal es el caso de muchas de las llamadas "reacciones civilistas" en la historia de América Latina. Un ejemplo evidente es el de Chile a partir de 1930 cuando la sociedad y los políticos reaccionaron a la militarización de la década previa lo que trajo aparejado una drástica reducción del gasto militar y una prolongada condena social a las fuerzas armadas (Nunn, 1976). Más recientemente se encuentra el caso de la Argentina en donde la experiencia colectiva con la última dictadura, que provocó un genocidio llevado a cabo por fuerzas militares y de seguridad gracias a una estructura estatal omnipresente, generó un profundo rechazo social hacia todo aquello que se asociara con el estado. Este antiestatismo de la sociedad argentina facilitó la implementación del programa neoliberal más radical de toda la región que comenzó a ser desmontado a partir de 2003, con la llegada a la presidencia de Néstor Kirchner. La etapa que comienza en el 2003 señala el retorno progresivo de un estado fuerte pero que esta vez comienza a ser percibido por la sociedad ya no en términos represivos sino como un actor que difunde el bienestar (Grugel; Riggirozzi 2007). No es casual entonces, que este contexto de reencuentro de la sociedad con el estado sentara las bases para un lento retorno de las cuestiones de la defensa y que se comenzara a pensar desde la política y la sociedad la necesidad de incorporarla a la estrategia de desarrollo nacional.

\section{COMENTARIOS FINALES}

Este trabajo ha explorado un nivel de análisis poco estudiando del concepto de defensa, su principal objetivo no ha sido la exhaustividad sino el de presentar una conceptualización que requiere de ulterior elaboración en la dirección de profundizar tanto la indagación sobre los fundamentos aquí analizados como así también de otros que no fueron contemplados. Asimismo, es necesaria mayor investigación empírica que permita comprender el impacto que los distintos fundamentos tienen sobre los medios y las definiciones de la defensa.

El artículo ha sido motivando en gran medida por la creciente difusión de lógicas de razonamiento que plantean una suerte de reduccionismo armamentista. En efecto, una proporción sustantiva de los debates sobre la defensa en la academia y en los medios de comunicación se concentra en 
la importancia de las armas y en la de otros medios de la defensa, desconociendo la relevancia de variables, como los fundamentos, que son las más decisivas a la hora de pensar y gestionar políticamente este tema. El diagnóstico sobre qué es lo principal y qué es lo secundario para la defensa de una nación no puede desconocer la existencia de aspectos fundamentales que son los que finalmente permiten dar cuenta de disimiles niveles de interés en tales cuestiones. Para ello es fundamental distinguir conceptualmente lo principal del epifenómeno.

Nada en este análisis apunta a menoscabar la importancia de las capacidades materiales, por el contrario, las armas son fundamentales y sin ellas no hay victoria posible en un conflicto. Asimismo, el nivel de coherencia entre la estrategia nacional, la estrategia militar y la táctica o doctrina militar es igualmente determinante para la defensa de una nación. Sin embargo, la dimensión de los fundamentos es decisiva en dos planos distintos. Cuando se trata de explicar las variaciones en los niveles de atención política sobre la defensa y cuando debe reflexionarse acerca de cómo promoverla de manera sostenible en el tiempo. La defensa de una nación se juega principalmente en sus fundamentos.

\section{BIBLIOGRAFÍA}

ACUÑA, M.; RUNZA, R. Hacia la modernización del sistema de defensa argentino. Buenos Aires: Altamira, 2006.

ANZELINI, L.; POCZYNOK, I. De la política militar a la política de defensa. El ciclo de planeamiento de la defensa nacional (2007-2011) como punto de inflexión. Perspectivas para la gestión, el Estado y las políticas públicas, v. 1, n. 2, p. 72-95, 2014.

ARREGUIN-TOFT, I. How the weak win wars: a theory of asymmetric conflict. New York and Cambridge: Cambridge University Press, 2005.

BATtAGLinO, J. Políticos y militares en los gobiernos de la nueva izquierda sudamericana. Política y Gobierno, v. XXII, n. 1, p. 3-43, primer semestre 2015.

BATTAGLinO, J. Auge, caída y retorno de la defensa en la Argentina. Foreign Affairs Latinoamérica, v. 13, n. 1, p. 32-39, enero-marzo 2013.

BIDDLE, S. Military power: explaining victory and defeat in modern battle. Princeton: Princeton University Press, 2006.

BUTFOY, A. Offence-defence theory and the security dilemma: the problem with marginalizing the context. Contemporary Security Policy, v. 18, n. 3, p. 38-58, 1997. 
BUZAN, B. Will the 'global war on terrorism' be the new cold war? International Affairs, v. 82, n. 6, p. 1101-1118, 2006.

BUZAN, B.; WEAVER, O. Regions and powers: the structure of international security. Cambridge: Cambridge University Press, 2003.

BUZAN, B.; WAEVER, O; WILDE, J. de. Security: a new framework for analysis. Boulder: Lynne Rienner, 1998.

CANELO, P. Consideraciones sobre la subordinación de las Fuerzas Armadas

argentinas durante los años noventa. In: PUCCIARELLI, A. (Coord.). Los años de Menem. La construcción del orden neoliberal. Buenos Aires: Siglo XXI, 2011.

CALLE, F. Rambo, versión sudamericana. El impacto regional del rearme de

Venezuela y Chile. Nueva Sociedad, v. 211, p. 13-21, 2007.

D’ARAUJO, M. C. Los militares y el desarrollo en América del Sur. In: DONADIO, M. (Comp.). La reconstrucción de la seguridad nacional. Defensa, democracia y cuestión militar en América Latina. Buenos Aires: Resdal-Prometeo, 2010.

DESCH, M. Threat Environments and Military Missions. In: DIAMOND, L.; PLATTNER, M. F. (Ed.). Civil-military relations and democracy. Baltimore and London: The Johns Hopkins University Press, p. 12-30, 1996.

DIAMINT, R. Control civil y Fuerzas Armadas en las nuevas democracias latinoamericanas. Buenos Aires: Nuevo Hacer, 1999.

DUARTE VILLA, R.; WEIFFEN, B. South American re-armament: from

balancing to symbolizing power. Contemporary Security Policy, v. 35, n. 1, p. 138-162, 2014.

DUBE, S.; PINZÓN, V. G. Las tareas contemporáneas de las fuerzas armadas latinoamericanas y la necesaria revisión de la teoría de la consolidación democrática. Estudos Ibero-Americanos, v. 38, n. 1, p. 119-143, 2012.

ESCUDÉ, C. El protectorado argentino y su indefensión actual: un análisis desde el realismo periférico. Trabajo presentado en el Congreso de FLACSO/ISA, Buenos Aires, julio de 2014.

EISSA, S. Lo que el neoliberalismo se llevó. Reconstruyendo la política de defensa argentina. Working Paper n. 40. Programa Defensa y Seguridad. Buenos Aires: Centro Argentino de Estudios Internacionales, 2010. 
FONTANA, A. La política militar en un contexto de transición: Argentina 1983-1989. Documento CEDES, v. 34, 1990.

FREDERIC, S. Los usos de la fuerza pública. Debates sobre militares y policías en las ciencias sociales de la democracia. Buenos Aires: Universidad Nacional de Quilmes, 2011.

GRAY, C. Strategic ideas and defense policy: the organizational nexus. In: BEAMONT, R.; MARTIN, E. (Ed.). War in the next decade. Lexington: University Press of Kentucky, 1974, p. 89-109.

GRUGEL, J.; RIGGIROZZI, M. P. The return of the state in Argentina. International Affairs, v. 83, n. 1, p. 87-107, 2007.

HUntingtOn, S. The soldier and the State. Cambridge Massachusetts: The Belknap Press, 1995.

HUSER, H. Argentine civil-military relations: from Alfonsín to Menem. Washington: National Defense University Press, 2002.

LAFERRIERE, G.; SOPRANO, G. El ejército y la política de defensa en la Argentina del siglo XXI. Rosario: Prohistoria, 2015.

LIEBER, K. War and the engineers. The primacy of politics over technology. Ithaca: Cornell University Press, 2005.

LÓPEZ, E. Ni la ceniza ni la gloria: actores, sistema político y cuestión militar en los años de Alfonsín. Buenos Aires: Universidad Nacional de Quilmes, 1994.

MANI, K. Military entrepreneurs: patterns in Latin America. Latin American Politics and Society, v. 53, n. 3, p. 25-55, 2011.

MURRAY, D.; VIOTTI, P. (Ed.). The defense policies of nations: a comparative study. Baltimore: John Hopkins University Press, 1994.

NUNN, F. M. The military in chilean history: essays on civil military relations, 1810-1973. Albuquerque: University of New Mexico, 1976.

ONEAL, J.; RUSSETT, B. The classical liberals were right: democracy, interdependence, and conflict 1950-1985. International Studies Quarterly, v. 41, n. 2, p. 267-293, 1997.

PION-BERLIN, D.; TRINKUNAS, H. Attention deficit: why politicians ignore defense policy in Latin America. Latin American Research Review, v. 42, n. 3, p. 76-100, 2007. 
PION-BERLIN, D.; ARCENEAUX, C. Decision-makers or decision-takers? Military missions and civilian control in democratic South America. Armed Forces E' Society, v. 26, p. 413-436, 2000.

RESDAL. Atlas comparativo de la defensa nacional. Buenos Aires: Resdal, 2015.

SCHEETZ, T.; PFURR, A.; GRATACOS, M. A. Manual de teoría de la gestión económica de las Fuerzas Armadas. Buenos Aires: Grupo Editor Latinoamericano, 2015.

SCHWELLER, R. Unanswered threats: a neoclassical realist theory of underbalancing. International Security, v. 29, n. 2, p. 159-201, fall 2004.

WALT, S. Alliance formation and the balance of world power. International Security, v. 9, p. 3-43, spring 1985.

WALTZ, K. Teoría de la política internacional. Buenos Aires: GEL, 1979.

WILSON, I.; FOREST, J. Handbook of defence politics. International and comparative perspectives. London and New York: Routledge, 2008. 


\section{NOTAS}

1. De un total de 53 trabajos sobre defensa en la Argentina publicados entre el 2000 y el 2014 ninguno hizo referencia a la dimensión de los fundamentos.

2. El "déficit de amenazas" puede ser definido como un escenario estratégico en el que la percepción o percepciones de amenaza a la defensa de un Estado desaparecen, o se debilitan considerablemente, y no son reemplazadas por otras nuevas. Un tratamiento detallado de este concepto puede encontrarse en Buzan (2006).

\section{FUNDAMENTOS OLVIDADOS DE LA POLÍTICA DE DEFENSA: REFLEXIONES A PARTIR DEL CASO ARGENTINO}

\section{RESUMEN}

Los estados destinan disímiles recursos materiales y simbólicos a su defensa nacional, es decir, le asignan distinta importancia a la misma. Este artículo propone uma conceptualización del concepto de defensa que incluye el nivel de los fundamentos los cuales permiten explicar las variaciones en el interés de los estados en este tema.

Palabras-clave: Defensa Nacional; Fundamentos Determinantes, Argentina.

\section{ABSTRACT}

The states allocate different material and symbolic resources to national defense, namely, assign different importance to the defense. This article proposes a conceptualization of the concept of defense, which includes the level of the fundamentals, which helps explain variations in the interest of the states in this area.

Key-words: National Defense; Determinat Grounds; Argentina. 